\title{
Inklusion im Sozialraum aus Sicht des Deutschen Vereins
}

\author{
Andreas Kuhn
}

I. Inklusion im Sozialraum aus Sicht des Deutschen Vereins

$\begin{array}{ll}\text { II. Soziale Inklusion } & 108\end{array}$

III. Barriere Wertschätzung? 111

IV. Literatur 114

\section{Inklusion im Sozialraum aus Sicht des Deutschen Vereins}

Die UN-Behindertenrechtskonvention hat dazu beigetragen, dass auch im Deutschen Verein das Thema Inklusion intensiv diskutiert wird und Papiere beschlossen wurden, die beschreiben, wie Inklusion zu verwirklichen ist: ein Diskussionspapier zu inklusiver Bildung, Eckpunkte für einen inklusiven Sozialraum und Empfehlungen zu einer örtlichen Teilhabeplanung für ein inklusives Gemeinwesen.

Auch zum Sozialraum hat sich der Deutsche Verein in praktischer Absicht geäußert ${ }^{1}$. Soziale Räume sind als - politisch oder statistisch definierte - Gebiete im Gemeinwesen zu verstehen, als Wohnquartiere und Stadtteile, in denen die soziale Integration der darin lebenden Bewohner und Bewohnerinnen stattfindet. Soziale Räume sind Gebiete im Gemeinwesen, die auch heute die Sozialisation, die persönliche Entwicklung, die Lebensbedingungen und die Lebensweise der darin aufwachsenden und lebenden Individuen beeinflussen ${ }^{2}$. In welchem Ausmaß, ist natürlich individuell unterschiedlich. Generell gilt: Je geringer die Mobilität, desto wichtiger ist der so definierte Sozialraum. Ein zweiter Einflussfaktor ist die residentielle Segregation insbesondere von ethnischreligiösen Gruppen.

Inklusion und Sozialraum in Zusammenhang gebracht haben die Arbeits- und Sozialminister auf ihrer Konferenz (ASMK) im November 2009. Die Konferenz stellt fest:

1 Und unterscheidet sich damit deutlich von den Debatten, die in Deutschland über den Begriff des Sozialen Raums geführt wurden; vgl. z.B. Kessel u.a. (Hrsg.), Handbuch Sozialraum, Wiesbaden 2005.

2 Eckpunkte des Deutschen Vereins zur sozialräumlichen Ausgestaltung kommunalen Handelns, NDV 9/2008, S. 377 ff. 
Die Weiterentwicklung der Eingliederungshilfe kann ihre volle Wirkung nur dann entfalten, wenn sie sozialräumlich unterstützt wird (Beschluss der Konferenz vom 25./26.11.2009), und belässt es bei dieser - plausiblen - Annahme. Bei der Weiterentwicklung der Eingliederungshilfe soll Inklusion als Leitidee umgesetzt werden, Hilfe soll personenzentriert sein (nicht einrichtungszentriert). Zum Auf- und Ausbau inklusiver Sozialräume sollen Handlungsstrategien erarbeitet werden, und die Weiterentwicklung der Eingliederungshilfe soll insgesamt kostenneutral sein.

In diesem Beitrag will ich aus der pragmatischen Sicht des Deutschen Vereins und aus Sicht der Stadtsoziologie sondieren, welche Bedeutung der Sozialraum für Inklusion hat und auf die These eingehen, dass inklusive Sozialräume nur in dem Maße verwirklicht werden können, wie den Menschen mit Behinderung ein ,positiver Wert“ zuerkannt wird.

\section{Soziale Inklusion}

Der Deutsche Verein definiert inklusiven Sozialraum als ein barrierefreies Lebensumfeld, das alle Menschen mit und ohne Behinderungen, alte und junge Menschen, Menschen mit oder ohne Migrationshintergrund selbstbestimmt gemeinsam nutzen und mitgestalten können. Nach dieser Definition sind die sachlichen und materiellen Bedingungen der entscheidende Faktor dafür, in welchem Maße ein Sozialraum Möglichkeiten für ein inklusives Leben bietet. Und Inklusion ist ein Thema für alle Menschen in einem Sozialraum, nicht nur für Menschen mit Behinderung. $\mathrm{Zu}$ einem inklusiven Sozialraum gehört auch, dass es dort Beratungs- und Unterstützungsstrukturen gibt und Netzwerke, die für alle Interessierten offen sind und zur Beteiligung einladen. Das ist nur möglich, wenn sich die Bewohner und Bewohnerinnen gegenseitig akzeptieren und Vielfalt in ihrem Lebensumfeld wertschätzen. Beteiligung an Planungs-, Gestaltungsund Entscheidungsprozessen ist ein weiteres wesentliches Merkmal inklusiver Sozialräume. In einem inklusiven Sozialraum werden Kinder- und Jugendliche mit und ohne Handicap von Anfang an gemeinsam von den zuständigen Institutionen gefördert und unterstützt.

Die ASMK hat in ihren Eckpunkten zur Weiterentwicklung der Eingliederungshilfe (Oktober 2009) ähnliche Elemente für einen inklusiven Sozialraum benannt. Die ASMK betont, dass es um wohnortnahe Strukturen und eine Vielfalt an Wohnformen geht und Unterstützung auch von ehrenamtlichen Strukturen und einem Hilfe-Mix getragen wird.

Die Empfehlungen für eine örtliche Teilhabeplanung stimmen mit diesen Elementen überein. Sie werden in drei Zielen für die Planung zusammengefasst: die Herstellung einer barrierefreien öffentlichen Infrastruktur, die Entwicklung eines an Inklusion ausgerichteten Hilfesystems und die Sensibilisierung der Öffentlichkeit für Diskriminierungsrisiken und damit verbunden die Schaffung eines Solidaritätsbewusstseins. 
Die ASMK und auch der Deutsche Verein benennen die wichtigen Elemente eines inklusiven Sozialraums. Die Papiere sind in dieser Hinsicht wahrscheinlich sehr instruktiv. Die Elemente werden jedoch nicht in eine Rangfolge gebracht. Natürlich könnten alle Elemente auch als „Barrieren“ für Inklusion interpretiert, sozusagen mit negativen Vorzeichen versehen werden. Aber die Papiere verzichten darauf, Barrieren für einen inklusiven Sozialraum so anzusprechen, wie das die Rehabilitationswissenschaft und Behindertenpädagogik doch sehr dezidiert tut, als Defizite eines Gemeinwesens oder einer Kommune, unter denen besonders Menschen mit Behinderung zu leiden haben. Obwohl Barrieren eindeutig ein wesentlicher Aspekt für Inklusion im Sozialraum sind, werden diese von der ASMK und auch vom Deutschen Verein nicht definiert, differenziert und erörtert. Das ist vielleicht ein deutliches Manko und müsste bei weiteren Empfehlungen aufgearbeitet werden. ${ }^{3}$ Auch dann sind „Barrieren“ jedoch ein analytisches Konzept und eine orientierende Kategorie, kein normatives Verdikt.

Sowohl die sozialpolitischen Akteure wie die Experten für Menschen mit Behinderung unterstellen, dass Sozialräume relevant sind für Inklusion ${ }^{4}$. Das ist - wie gesagt plausibel, aber es wäre natürlich gut zu wissen, welche Strukturen eines Sozialraums und welche Mechanismen in Sozialräumen es sind, die Inklusion befördern, erschweren oder verhindern. Und es könnte angezeigt sein, den Begriff „Inklusion“ zu differenzieren, um Zusammenhänge mit dem Sozialraum zu beschreiben und zu analysieren. Genau dieser Aufgabe hat sich ein Forschungsprojekt im Auftrag des Bundesarbeitsministeriums gewidmet, das Möglichkeiten der verbesserten sozialen Inklusion in der Wohnumgebung untersucht hat. 5

Die Bezeichnung „Sozialraum“ wird oft synonym mit „Stadtteil“, „Ortsteil“, „Quartier“ oder „Wohnumgebung“" verwendet. Damit gemeint sind „kleinräumige Einheiten“, die durch den geografischen Raum wie durch die soziale Zusammensetzung der Bewohnerschaft geprägt werden. So verstanden, lassen sich drei Eigenschaften bzw. Mechanismen in Sozialräumen feststellen, die Inklusion maßgeblich beeinflussen:

- die Infrastrukturausstattung und -qualität (insbesondere Bildungseinrichtungen und das Gesundheitssystem, auch Sport- und Freizeiteinrichtungen sowie soziale Dienste), die Verkehrssituation und bauliche Struktur. Vor allem die Schulen

3 Eine sehr gute fachliche Grundlage dafür bieten die Ergebnisse des Sondergutachtens „Barrierefreie Stadtquartiere“, das im ExWoSt-Forschungsfeld „Innovationen für familien- und altengerechte Stadtquartiere" des BMVBS entstanden ist und im August 2011 veröffentlicht wurde; vgl. Bauer/Böcker/Rohlwing/Dähner, Barrierefreie Stadtquartiere, ExWoSt-Sondergutachten im Auftrag des Bundesministeriums für Verkehr, Bau und Stadtentwicklung, Berlin 2011.

4 Und den politisch-pragmatischen Papieren ist nachzusehen, dass Inklusion stark tautologisch positioniert wird, wenig systematisch; dass z.B. ein an Inklusion ausgerichtetes Hilfesystem ein inklusives Gemeinwesen auszeichnet.

5 Häussermann/Schwarze/Jaedicke/Bär/Bugenhagen, Möglichkeiten der verbesserten sozialen Inklusion in der Wohnumgebung, Forschungsprojekt im Auftrag des Bundesministeriums für Arbeit und Sozialordnung, Schlussbericht, Berlin 2010. 
verstärken oder kompensieren Benachteiligungen, die sich aus den sozialen Beziehungen (s.u.) ergeben;

- soziale Beziehungen, die das informelle Lernen (insbesondere über Peers) und die sozialen Netzwerke (oft über die Verwandtschaft) bestimmen. Soziale Beziehungen vermitteln Normen und Vorbilder und beeinflussen darüber das Denken und Verhalten in einem Sozialraum (,kollektive Sozialisation“) ${ }^{6}$. Je nachdem wie homogen oder heterogen ein soziales Netz ist, schränkt dieses Erfahrungs- und Kooperationsmöglichkeiten für den Einzelnen ein oder bietet ihm zusätzliche Chancen (Opportunities);

- Teilhabe an Veränderungen des Sozialraums, insbesondere an Entscheidungen der Politik und Verwaltung über die Quartiersentwicklung (Partizipation). Genau solche Partizipation soll Örtliche Teilhabeplanung organisieren und durchsetzen. Wenn das gelingt und die Erkenntnisse der Stadtsoziologie zutreffen, befördert örtliche Teilhabeplanung ganz erheblich die Inklusion in Sozialräumen.

Soziale Inklusion, um die es hier geht, hat also mehrere Dimensionen: eine institutionelle, eine kulturelle, eine politische und eine soziale, darüber hinaus eine ökonomische Dimension ${ }^{7}$. D.h. eine Person kann z.B. aus dem Erwerbssystem ausgeschlossen, ihre kulturelle und soziale Inklusion aber intakt sein. Der heutige Arbeitsmarkt (als Kern der ökonomischen Dimension sozialer Inklusion) ist keine Eigenschaft des Quartiers, sondern ein makrostruktureller Faktor für Inklusion und deshalb für die Sozialraumperspektive eine externe Variable.

Quartiere haben Effekte für die soziale Inklusion, jedoch nur in dem Maße wie die räumliche Nachbarschaft auch die soziale Nachbarschaft ist, d.h. desto mehr sich die sozialen Kontakte auf die nachbarschaftliche Umgebung beschränken, wenn also das Quartier der relevante Sozialraum ist ${ }^{8}$. Die Wirkungen sind somit bei verschiedenen Gruppen der Bewohnerschaft unterschiedlich ausgeprägt. Das hängt auch von individuellen Eigenschaften (Vulnerabilität/Resilienz) ab. Insgesamt sind die Wirkungen gegenüber der sozialen Herkunft und der Einbettung in die Familie nachrangig. Das ist die Quintessenz stadtsoziologischer Forschung zur sozialen Inklusion.

Was heißt das für die Inklusion von Menschen mit Behinderung? Prinzipiell dasselbe wie für alle anderen Menschen in einem Sozialraum auch. Es kommt darauf an, wie sie sich in den einzelnen Sphären der Inklusion bewegen und diese für ihre persönliche Entwicklung nutzen können und welche persönlichen Voraussetzungen und Erfahrun-

6 Häussermann (Fußn. 5), weisen darauf hin, dass auch die Außenwahrnehmung eines Quartiers das Denken und Verhalten der darin lebenden Menschen beeinflusst. Die Stigmatisierung eines Quartiers trägt dazu bei, dass Verantwortung und Bindung an die eigene Lebensumwelt deutlich abnehmen, S. 14-16.

7 Diese analytische Differenzierung von Inklusion wird hier angewendet ohne der Luhmannschen Systemtheorie zu folgen.

8 Dementsprechend handelt es sich bei einem Stadtteil dann um einen Sozialraum, wenn von der sozialen Zusammensetzung dieses Stadtteils Wirkungen auf das Denken und Handeln der Bewohner ausgehen, vgl. Häussermann (Fußn. 5), S. 3. 
gen sie dafür mitbringen. Für Menschen mit Behinderung haben die materielle Infrastruktur und die sozialen Dienste sicher eine relativ größere Bedeutung als für Menschen ohne Behinderung, und bei den sozialen Beziehungen könnte der Aspekt der Unterstützung eine relativ größere Bedeutung haben als bei Menschen ohne Behinderung. Aber auch diese Bedeutungen sind individuell ausgeprägt und abhängig von persönlichen Dispositionen und Interessen.

\section{Barriere Wertschätzung?}

Die Heilpädagogik und Rehabilitationswissenschaft fokussiert im Sozialraum die Menschen ohne Behinderung (ohne diese zu benennen). Aus ihrer Sicht ist ein „kultureller Wandel“" erforderlich, wenn inklusive Sozialräume oder Lebensräume auch für Menschen mit Behinderung geschaffen werden sollen. Wesentlicher Inhalt dieses kulturellen Wandels ist die Wertschätzung von Menschen mit Behinderung. Es brauche die „Zuerkennung eines positiven Wertes behinderter Menschen“. Das Fehlen dieser Wertschätzung sei das zentrale Hindernis, um Inklusion im Sozialraum zu verwirklichen. ${ }^{9}$

Aus der Perspektive der dargestellten sozialen Inklusion stellt sich die Frage: warum ist eine besondere Wertschätzung von Menschen mit Behinderung erforderlich, damit inklusive Sozialräume entstehen können? Warum reicht es nicht, dass Menschen mit Behinderungen (mit ihren Eigenarten) genauso akzeptiert werden wie Menschen mit anderen Eigenarten auch?

Der Deutsche Verein hat sich - wie gesagt - nicht dazu geäußert, welche Bedeutung die einzelnen Elemente für die Schaffung eines inklusiven Sozialraums haben. Die drei Zieldimensionen Barrieren, Unterstützungssystem und Diskriminierungsfreiheit/Solidarität stehen gleichberechtigt nebeneinander. Das dritte Ziel thematisiert den Aspekt Wertschätzung, allerdings nicht normativ-ethisch, wie die These oben, sondern pragmatisch, so wie es eine Handlungsstrategie erfordert. Und wie alle anderen Elemente auch, wird dieses Ziel als Aufgabe oder Herausforderung verstanden, nicht als Hindernis. Das ist sozusagen eine normativ-pragmatische Herangehensweise an den inklusiven Sozialraum.

Reduzieren wir den ethischen Anspruch an die Menschen im Sozialraum und übersetzen Wertschätzung mit Akzeptanz oder Anerkennung, dann können wir - einigermaßen - aussagekräftige empirische Forschung befragen. Dazu gehört vor allem die „Berliner Kundenstudie“. Diese ermittelte, dass $80 \%$ der befragten Menschen mit Behinderung ihre Nachbarn als freundlich bezeichnen. ${ }^{10}$ Die Studie zum Ambulant betreuten

9 Diese These hat Dederich auf der Fachtagung vorgetragen, aus der diese Publikation entstanden ist, siehe S. 61 in diesem Band.

10 Seifert, Kundenstudie. Bedarf an Dienstleistungen zur Unterstützung des Wohnens von Menschen mit Behinderung, Berlin 2010, S.129. 
Wohnen in Bayern von Frau Hanslmeier-Prockl 11, die hohe Anforderungen an Nachbarn stellt (,erweitertes Netzwerk"), muss zwar vorsichtig aufgenommen werden, weil die Aussagen zur Nachbarschaft nicht eindeutig sind, aber es lässt sich folgern, dass Akzeptanz im Umgang miteinander ein verbreiteter Umgangsmodus zwischen Menschen mit und ohne Behinderungen ist. Frau Hanslmeier-Prockl stellt fest, dass Reaktionen der Nachbarn eher positiv ausfallen 12 und „dass nicht von einer grundsätzlichen Ablehnung von Menschen mit Behinderung gesprochen werden kann ${ }^{13}$ “ Vergleicht man diese Ergebnisse mit dem Fazit stadtsoziologischer Forschung, wird deutlich, dass hier ganz „,normale“ Nachbarschaften untersucht wurden: „Allein aus lokaler Nähe entsteht nur ein geringes Interesse aneinander - Hauptsache, man stört sich nicht““.14

Von diesen Erkenntnissen lässt sich eine Studie zur „Teilhabe von Menschen mit geistiger Behinderung am Leben in der Kommune“ leiten. ${ }^{15}$ Die Autorinnen betonen, dass Menschen mit geistiger Behinderung ein so normales Leben wie möglich führen müssen und das tun sollen, was andere Bürgerinnen und Bürger auch tun: „Angebote machen und Angebote wahrnehmen. Sich selbstverständlich und sichtbar als Helferin beim Stadtfest, als Musiker im Verein und als Kundin beim Bäcker bewegen“, anerkannte und sozial geachtete Rollen ausüben. Dann entstehen „gemeinsame Sozialräume"16. Soziale Inklusion ist nach Ihrer Auffassung am wahrscheinlichsten, wenn Menschen mit Behinderung die materielle Infrastruktur einer Kommune in geeigneter Qualität zugänglich ist und von diesen genutzt wird (insbesondere öffentlicher Personennahverkehr und Institutionen der Bildung und Arbeit) ${ }^{17}$. „Inklusionsbereitschaft" der Bürgerinnen und Bürger ist aus soziologischer Sicht nicht die Voraussetzung dafür, dass Menschen mit Behinderung ebenso Bürgerinnen und Bürger in einem Sozialraum sein oder werden können. Aus pragmatischer Sicht kann darauf verzichtet werden, die Einstellung von Personen mit solchen Kategorien zu kennzeichnen. Viel wichtiger ist es, dass möglichst viele Bewohnerinnen und Bewohner in einem Sozialraum konkrete positive - Erfahrungen im Umgang miteinander machen. Damit entstehen oder verstärken sich Haltungen und Einstellungen, in diesem Fall mit hoher Wahrscheinlichkeit solche, die Inklusion befördern.

Die Studien zur sog. „gruppenbezogenen Menschenfeindlichkeit“ zeigen, dass Menschen mit Behinderung unter den „Minderheiten“ in Deutschland mit Abstand am meis-

11 Hanslmeier-Prockl, Teilhabe von Menschen mit geistiger Behinderung. Empirische Studie zu Bedingungen der Teilhabe im Ambulant betreuten Wohnen in Bayern, Bad Heilbrunn 2009.

12 Hanslmeier-Prockl (Fußn. 11), S. 93.

13 Hanslmeier-Prockl (Fußn. 11), S. 195.

14 Häussermann/Siebel, Stadtsoziologie. Eine Einführung, Frankfurt/New York 2004, S. 112.

15 Erhadt/Grüber, Teilhabe von Menschen mit geistiger Behinderung am Leben in der Kommune, Freiburg im Breisgau 2011.

16 Erhadt/Grüber (Fußn. 15), S. 125.

17 Erhadt/Grüber (Fußn. 15), S. 58. 
ten akzeptiert werden ${ }^{18}$. Nur etwa sieben Prozent der Befragten stellen den Umfang der Unterstützung und die Ausprägung der Forderungen von Menschen mit Behinderungen in Frage. Es ist schon gewagt, von diesen Umfrageergebnissen darauf zu schließen, dass diese sieben Prozent einen Menschen mit Behinderung als Nachbarn nicht akzeptieren würden, nicht nachvollziehbar ist es, wie von Umfrageergebnissen mit solchen Items auf „Abwertung von Menschen mit Behinderung“ geschlossen werden kann. Mit solchem Herangehen könnten die kommunalen Spitzenverbände in Deutschland verdächtigt werden, Menschen mit Behinderung abwerten zu wollen, weil sie seit Jahren darüber diskutieren, wie die Ausgaben für Menschen mit Behinderung eingegrenzt werden können.

Ich bin ziemlich sicher, dass in einer Soziologie der Inklusion Akzeptanz und Anerkennung grundlegende Kategorien und Konzepte sein müssen und auf eine Ethik der Wertschätzung verzichtet werden kann, um zu verstehen und zu befördern, wie „Menschen mit und ohne Behinderung, alte und junge Menschen, mit und ohne Migrationshintergrund gemeinsam Lebensräume nutzen und gestalten" können - um noch einmal den Deutschen Verein zu zitieren. ${ }^{19}$

Wenn es stimmt, dass ,,sich begegnende Personen dazu genötigt sind, auch ihr soziales Gegenüber in einer bestimmten Weise anzuerkennen, um sich in dessen Reaktion selbst anerkannt zu finden - die Anerkennung des Gegenüber wird zur Bedingung des eigenen Anerkannt-Seins" ${ }^{20}$, dann ergibt sich für die soziale Inklusion von Menschen mit Behinderung tatsächlich eine spezifische Frage: wie ist Reziprozität mit Menschen ohne Behinderung möglich? Ist Reziprozität in solchen zwischenmenschlichen Beziehungen überhaupt möglich?

18 Vgl. zu dazu und zum Folgenden http://www.uni-bielefeld.de/ikg/projekte/GMF/index.htm (Zugriff am 09.08.2012).

19 Eckpunkte des Deutschen Vereins für einen inklusiven Sozialraum, NDV 1/2012, S. $1 \mathrm{ff}$.

20 Honneth, Verwilderungen. Kampf um Anerkennung im frühen 21. Jahrhundert, Aus Politik und Zeitgeschehen 1-2/2011, S. 37 f. 


\section{Literatur}

Bauer, U./Böcker, M./Rohlwing, S./Dähner, S., Barrierefreie Stadtquartiere, ExWoSt-Sondergutachten im Auftrag des Bundesministeriums für Verkehr, Bau und Stadtentwicklung, Endbericht, Berlin 2011.

Eckpunkte des Deutschen Vereins zur sozialräumlichen Ausgestaltung kommunalen Handelns, NDV 9/2008, S. 377 ff.

Eckpunkte des Deutschen Vereins für einen inklusiven Sozialraum, NDV 1/2012, S. 15 ff.

Erhadt, K./Grüber, K., Teilhabe von Menschen mit geistiger Behinderung am Leben in der Kommune, Freiburg im Breisgau 2011.

Erstes Diskussionspapier des Deutschen Vereins zu inklusiver Bildung, NDV 5/2011, S. 197 ff.

Empfehlungen zur örtlichen Teilhabeplanung für ein inklusives Gemeinwesen, NDV 6/2012, S. $286 \mathrm{ff}$.

Hanslmeier-Prockl, G., Teilhabe von Menschen mit geistiger Behinderung. Empirische Studie zu Bedingungen der Teilhabe im Ambulant betreuten Wohnen in Bayern, Bad Heilbrunn 2009.

Häussermann, H./Schwarze, K./Jaedicke, W./Bär, G./Bugenhagen, I., Möglichkeiten der verbesserten sozialen Inklusion in der Wohnumgebung, Forschungsprojekt im Auftrag des Bundesministeriums für Arbeit und Sozialordnung, Schlussbericht, Berlin 2010.

Häussermann, H./Siebel, W., Stadtsoziologie. Eine Einführung, Frankfurt/New York 2004.

Honneth, A., Verwilderungen. Kampf um Anerkennung im frühen 21. Jahrhundert, Aus Politik und Zeitgeschehen 1-2/2011, S. 37 ff.

Seifert, M., Kundenstudie. Bedarf an Dienstleistungen zur Unterstützung des Wohnens von Menschen mit Behinderung, Berlin 2010. 